UDC 811.111'22

\title{
THE LINGUISTIC VERIFICATION OF LACUNAE BY THE MODERN LACUNOLOGY STUDIES
}

\author{
Tetiana Anokhina, \\ Germanic and Finno-Ugric Philology Department, \\ Kyiv National Linguistic University, \\ Kyiv, Ukraine \\ e-mail: anokhina_mail@yahoo.com \\ Received February, 22, 2018; Accepted April, 30, 2018
}

\begin{abstract}
Lacunology is a new linguistic discipline which is developing today due to new approaches oriented towards research, analysis and experiment. Lacunology studies pertain to an international dimension supporting its status as a new linguistic discipline. Linguistic research has been compiling the terms of lacunology into the registry of lacunicon. Lacunology deals with sociolinguistics, nonverbal semiotics, corpus linguistics and other related linguistic areas. Lacunology focuses on the interpretation of empty spaces in contrasting languages, zero verbalization in written and oral discourse, and null transfer, or the explicit substitutes for the lacunar originals. This article gives the current status of lacunology studies where lacunae are regarded as results of change, innovation, substitution and transfer. The paper has revealed many lacunar linguistic phenomena which can be identified and found in texts and corpus data if lacunae are seen as rare words or hapaxes. It is important to differentiate communicative lacunae (lacunae in speech), paradigmatic lacunae (lacunae in language), and conceptual lacunae (lacunae of cognition). The methods to render lacunae in translation are revealed by the current linguistic study. The methods have been discussed by researchers.
\end{abstract}

Key words: lacunae, gaps, lacunicon, hapaxes, lacunology studies.

\section{Introduction}

Lacunology is a new linguistic discipline, which has its own fixed and non-fixed inclinations and terms. The category of lacunarity has the characteristics of "cyclical repetition", which reflects the process of reduction and the simplification of languages in a scientific worldview. The constant developement of lacunicon terminology is in the state of an on-going and renewal. It makes sense to research the category of lacunarity and its appropriate terms. The object of this article has revealed many lacunar linguistic phenomena that can be identified and found in texts and corpus data - the subject of the article is when lacunae are seen as rare words or hapaxes.

The Lacunology studies were initiated by the French-Canadian School of Lacunology while embracing translation studies. The Lacunology Studies are devoted to empty places in the structure of contrasting languages and the ways of zero verbalization in written and oral discourse. The empty categor of "lacunarity" has been studied by the Canadian translators Jean-Paul Vinay (1910-1999) and Jean Darbelnet (1904-1990) and their followers. 
The Lacunology Study has "lacuna" as the basic term and its status was verified by the new linguistic science - lacunology. The root of the word lacuna is frequented in many lexical units: lacuna, lacune, lacunar, lacunarity, lacunicon, lacunology. Lacuna is of Roman origin (Greek - Latin) and is semantically charged as "lake", "pond", "swamp", "uncultivated land", "pit", and "cave". These words area of landscape topography. Later on, lacuna terms joined different domains of people's activities, but today lacunicon is fortunate to be included in a terminological system of lacunology (at the end of the 20th and the beginning of the 21st century).

Lacuna is often seen as lacking a unit, zero verbal nomination, absent meaning, nonexistent concept in one language, culture and society which has no corresponding equivalent. Lacunae are systematized by linguists according to the following parameters: lacunae in language (the results of reduction, elision); lacunae in speech (caused by cognitive disonance resulting in pauses); and extralinguistic nature (aphasia).

In Translation Studies lacuna are units that have a single-word nomination in another language. Lacunae are empty spaces in the system of language and vague concepts. Modern linguistics, translation studies and nonverbal semiotics have given birth to a new science that has become viral today - lacunology, the science which studies pauses in speech, cultural and linguistic gaps, lacunae of cognition, and zero transfer. The expansion of many various methods and disciplines interference resulted in the development of lacunology studies.

Lacuna is a unique phenomenon which mirrors zero reflection of nonequivalent vocabulary. Lacunae are quasi-comparable units that can refer to various referents of ethnic cultures. The phenomenon of the lack of specific elements in the culture of one ethnic group against another in English termed as "gap". Cultural or linguistic facts can cause gaps or broad interpretation. Ethnographic specifics must be fully rendered in the target culture. The implicit gaps are results of the loss of some background information.

Lacuna is a signal of the specificity of a particular linguistic community compared to another. By gaps, or lacunae, linguists understand: the absence of a semantic structure of the word that would provide the designated object, or phenomenon. There in no a unonimous term for this phenomenon. Lacuna can also be termed as lacunema. Fragmentary presentation of the information in a sentence, which is reflected in the incompleteness of the composition of the subject and the predicate is traditionally treated as the omission of minor members of the sentence. The term gap is also used. The reduction of the syntactic structure is typically regarded as ellipsis.

\section{Lacunae in language, speech and cognition}

There are different layers of lacunarity. The paradigmatic lacunarity can be illustrated by grammatical categories, such as the category of the article in English, or the category of gender in Ukrainian that emerge as zero in contrast. This phenomenon is considered to be a throwback to the synthetic background of the Ukrainian language and analytic background of the English language. The analytic 
design of English, its simplified wording stays lacunar for the Slavic speech model. Also, the word order of the English Language is standard in Slavic Languages.

The term linguistic lacunicon as a component of the structure of linguistic identity was suggested by Yuriy Sorokin (Sorokin, 1988); and later the term lacunicon became "a mechanism that controls the filtering of all incoming information" (Glazacheva, 2005: 33). The lacunicon is a terminologic registry, which includes linguistic units correlating with the semantics of lacunarity, absence, reduction, compression, truncation, and elision. These gaps are empty, implicit, compressed elements of language systems, voids of communication, speech emptiness (e.g., pauses, silence, the silent effect), and the lacunae of cognition.

The linguistic lacunicon includes the linguistic terms within grammatical, lexical, phonetic and syntactic clusters (the paradigmatic gaps), and the terms in respect with communicative and neuro-physiological gaps in speech (the syntagmatic gaps). Also, terms denoting the lack of background or specifically oriented knowledge regarding the linguistic lacunicon which is related to the semantics of decoding (the cognitive gaps) can also be used.

The syntagmatic cluster of the register of the linguistic terms of lacunicon has been formed by the following terms: compression, summarization, rhetorical question, interrogation. There are some culture-oriented terms of the lacunicon: ethos, ethnonyms, nationally biased lexicon, and xenomyms. The cognitive terms are adherent to the cluster of the linguistic lacunicon, among them there are such terms as: knowledge lacuna/gap, cognitive lacuna/gap, semantic lacuna/gap, narrowing of meaning, restriction of meaning, specification of meaning, and syncretism. The ethnographic elements are considered by linguists as gaps of transfer to another culture: that are not easily translated, so they are rendered with a phrase, not by a word (Anokhina, 2015: 10).

The semantic vector of the lacunicon is oriented towards a terminological development, producing such linguistic terms in the scientific worldview as: a reduced form; altered/transformed meaning; changed/modified meaning; faulty interpretation; incorrect interpretation; wrong interpretation/misinterpretation; erroneous interpretation; nonproductive substitution; and discrepancy/variance/mismatch of the multilingual products correlates.

The lexical and grammatical change in certain combinations differ in perception with native speakers. For example, not incidentally the German combination of "zärtliches Schweigen", Ukr. "ласкава тиша" is translated as "Gentle silence". "Soft and mute" is a rare combination, author's epithets are unexpected with hidden comparisons that attract attention of the reader, so the translator tends to artificially save the reproducibility of the collocation.

Creating sequels of books and numerous screen versions provide lacunar prototypical copies, relics or artifact parodies, such as "Casino Royale" (1967). New versions are not worthy without comparison to older ones. Translations and a new film are always intertextual. The intertextuality is the "right hand" of semantic lacunarity. Artifact, where you can find the gaps, is incomplete with respect to its real or virtual prototype. When something is lost in translation, it must be the identity of the original. 
Taxonomy of lacunar mentalese include the bottom taxon nothing and a top taxon everything denoting no distinctions (Frank, 2006: 32). Both everything and nothing in Boolean logic has no distinctions, thus lacunar taxons are taxons that have vague distinctions, or no distinctions at all. According to Frank (2006) a taxon is described as two sets of Boolean distinctions: the set of the affirmed and the set of negated distinctions ( $\mathrm{D}=\{$ True. False $\}$ ) (ibid 2006: 32). The word concept describes a unit in the mental realm, something in our mind (Frank, 2006: 28). The taxons of a cognitive endozone can be highly figurative, cf.: heavy thoughts, green ideas, flying proposals (Frank, 2006: 37).

If analogy to the bifurcation processes is applicable to word building in multi word units, before splitting a neo, the virtual would-be element goes back to branch of earlier forms and other language potentialities and have resulted in the top choice. In a synergetic approach, the bifurcation firstly goes to the old before creating something new like the Celtic stone (also known as rattle back, anagyre, celt, rebellious celt, rattlerock, spin bar, Robinson Reverser, wobble stone, wobblestone spins in the opposite direction, becomes unstable, "rattles" to a stop and reverses its spin to the preferred direction. Burt (2003) drew this analogy, based on case studies, with the biological concept of epigenetic change demonstrating how the epigenetic change "grows new from the seeds of the old" positing the use of a transitional object, "a mental construct that establishes continuity between the proposed changed situation and the past" (Burt, 2003: 391).

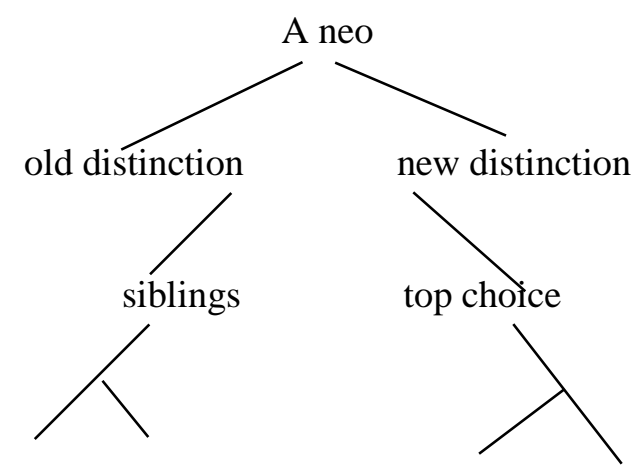

Figure 1. New vs. old distinction: new meaning, siblings, top choice

\section{Communicative lacunae: grammatical, constituent, discourse, conceptual ellipsis}

The "communicative gap" is seen as zero externalization in speech, which is correlated with a lack of knowledge of specific realities, ethnonyms or other specifics thus resulting in pauses, and then in cognitive dissonance. Gaps are opposed to nonequivalent vocabulary. A gap is a prototype of a zero element. In translation, gaps emerge while the unknown information transfers through the thesaurus of the personality - the personal AVN (the associative-verbal network). Gaps are fixed at 
the time of comparison and reconstruction of the foreign language and an unfamiliar culture. Making comparisons to other languages, we aim at finding lacunae in contrasting elements. Nonverbal gaps are eliminated both verbally and non-verbally over time, and have a protracted temporal way that can be measured in years.

The ellipsis occur in speech and in writing. The planned ellipsis usually correspond to pauses and hesitation phenomena in speech forming syntactic or conceptual lacunae. There are many forms of ellipses researched in different manners. Mostly, the grammatical ellipsis was analyzed by grammar researchers, including The Generative Grammar School, as the "Ellipsis as grammatical indeterminacy" (Shopen, 1973: 65). The researchers were connected to the phonetical ellipsis, other studied graphical exteriorization of the silence effect (Anokhina, 2015: 9). There are some researchers of ellipses viewed under an interrogative category as dialogue markers. The need for conceptual ellipsis has been strongly emphasized by many researchers including Malcolm, Lehnert and McDonald (Malcolm, Wendy \& McDonald, 2017: 5). The most vivid illustrations of semantic ellipsis can be given by postmodern writers' prose or semantic analysis of the reduced paragraphs, texts and meanings. There are some major ellipsis types below: functional, constituent, discourse and conceptual ellipses. The functional ellipses where constituents play the role of arguments without predicates to govern them can be demonstrated in the followig pattern: Hey Mike. What? Ann's coat. O.K.

The constituent ellipses where predicates are expressed without all their arguments are shown in the pattern: Hello Henry. What happened? Bobby refused. What will we do now?

The discourse ellipsis can be found in the discourse grammar has many elliptical utterances, e.g., "Yes, I do". This form of utterance requires some kind of linguistic context for intelligibility. These structures should be specifically marked to just this effect (Anokhina, 2015: 5).

The conceptual ellipsis can be illustrated in a simple story (as authors claim) and in précis of a story named COMSYS (Japanese Construction Company). The following simple story précis will demonstrate the general phenomenon of the conceptual ellipsis given parallel to the story in the table below, cf.:

\section{THE COMSYS STORY}

John and Mike were competing for the same job at IBM. John got the job and Mike decided to start his own consulting firm. COMSYS. Within three years, COMSYS was flourishing. By that time, John had become dissatisfied with IBM so he asked Mike for a job. Mike spitefully turned him down.

\section{Précis:}

(a) "John wanted to work for IBM and so did Mike. They hired John and did not hire Mike".

(b) "Both John and Mike wanted to work for IBM, but they (only) hired John".

(c) "Mike wanted to work for IBM, but they hired John".

The analysis of this text in terms of plot units has "Competition" as a central unit in the graph (conceptual idea), which would make it a candidate basis for a summary of the story (Anokhina, 2015: 6). The following texts have realized the concept "Competition" in a précis model, preferences dictated by conceptual ellipsis 
aside. Discourse fluency effects are also treated as verb phrase deletion or pronominalization and can be regarded as the process or fact of using a pronoun instead of another sentence constituent, such as a noun or noun phrase. The $a, b, c$ choices vary according to how much of the content of the Competition unit they explicitly express.

An ellipsis (or lacuna in speech) is conveyed as the reader feels that there is something lacking. Though the key events are described the lacuna exists. Some enigma is hidden for the reader to derive later on. In other words, some information that the audience can infer on their own is intentionally left out (the conceptual ellipsis).

Conceptual ellipses usually occur when a story ends open, for instance as in "City of Glass" by Paul Auster where the presupposed detective genre is a postmodern story with an open end (Auster, 1986: 131). The change of genre is seen as genre ellipsis and must be collaging in the eclectic postmodern. In texts, some presupposed parts (such as the end of the story) of text are excluded which can cause indeterminacy. The core eleme nts may not have been preserved, or lost (the main hero lost in the postmodern story) in the story and concepts are changed (The CONCEPT - SOLVE $A$ RIDDLE of a detective genre changed to the CONCEPT - WHO AM I? in the post modern interpretation). The conceptual lacunae in cognition and textual ellipses are waiting for their interpretation and transfer.

The ellipsis, either planned or spontaneous, should be duly comprehended in order to be transferred into a secondary unit of translation by means of the functional ellipsis, the constituent ellipsis, the discourse ellipsis, or by the conceptual ellipsis in compressed translations, such as annotations or resumes. As we understand by lacunae in spontaneous speech pauses, the research of planned ellipses is highly desired to discuss and illustrate syntactic lacunae and conceptual lacunae differences.

\section{Lacuna as result as zero transfer in translation studies}

The problem of limited, or vague understanding as part of the source text, can be solved by quite different translation strategies and solutions. Referring to Anthony Pym's (Pym, 1993) the understanding of transcultural relations, there are three strategies for lacunae elimination: transposition, substitution, or modulation, to translate the textual lacunae (Pym, 1993: 34). The specifics of the social lacunae in terms of Anthony Pym can be rendered, or some part of the original can be transmitted, but the remainder should be left in the commentary of the original with a footnote, just not translated. Some elements of a culture cannot be translated. These gaps, or empty spaces in translation, are called the cultural specifics that can be lost in translation.

At the current moment, the lacunae theory is often associated with translation studies in which lacunae are interpreted as 'translation gaps`caused by irrelevance in the target text of translation where the specific, or vague information, is often substituted by "zero equivalents". The ways to eliminate lacunae are associated with such terms as non-adequacy of translation and methods to hide lacunae are described by the terminological such synonymic notions as: (i) "naturalizing" ("domestication") and (ii) "estranging" ("foreignization") (Anokhina, 2013b: 39). 
When lacunae stay lacunae after translation people call it foreignization (e.g., borsch, as Ukrainian borsch is a soup of Ukrainian origin that is popular in many Eastern and Central European cuisines, including those of Poland, Russia, Ukraine and Belarus. In most of these countries, it is made with beetroot as the main ingredient. In some countries, tomato is used as the main ingredient, while beetroot acts as a secondary ingredient), the adding technique usually accompanies foreignization, for the word is usually transcribed, or transliterated, preserving the original pronunciation and stressing the uniqueness of the phenomenon. Other translators argue that domestication is better, working from multilingual source texts, and doing translations which are very easily comprehended, cf. green borscht is sorrel soup. The success of a translation may be at the same time a failure for many traditional and not-sotraditional ways of looking at translation (Pym, 1996: 207).

All in all, the choice seems to be wavering between two main strategies: foreignization or naturalization/domestication - between evidencing the fact that the concept in question is alien to the target culture and enhancing the acceptability of the target text for the readers, but at the same time eliminating its cultural specifics.

Lacunae usually have zero exteriorization. The content of the lacunae is filled by borrowing, transliteration, or comparison: Eng. gastropub - Ukr. "zacmponaб". The gap can be compensated by an equivalent replacement, Cf.: Eng. earworm, Ukr. набридлива мелодія (obsessive melody). Lacunar neologic elements at first are transmitted descriptively, and later - with the help of equivalents, e.g.: Eng. man cave (a room or space (as in a basement) designed according to the taste of the man of the house to be used as his personal area for hobbies and leisure activities) may be translated into Ukrainian as "барлога/барліг" or "святе місие" differently depending on the context and connotations meaning "Cage" or "Holy place". While the transfer of information certain difficulties arise in translation (horror hows) causing lacunae.

The lacunarity is syncretically associated with the concept of "implicitness", it refers to the interpretation of the multi-vectored nature of semantic gaps in indirect and additional interpretations. The lacunarity is embodied in the uniqueness and idiomacy of the national culture, the mentality of people. In the "mirror" of a culture, we see only a partial reflection, the clarity of which depends on the quality of the elimination or compensation processes. To transfer every specific element from one text to another is important for students and future translators to form a translator's linguistic and cultural outlook, derived from a scientific and naive outlook of the world (for example, knowledge of industrial terminology and names of everyday objects). The formed knowledge intertwines, creating a holistic view of the world. A different interpretation and consequently its translation, or retranslation of the text, arises from the thesaurus volume, thus resulting in gaps of functional ambiguity and the ambiguity of interpretation.

An example of an ambiguous interpretation can be fixed in prepared speech, or in spontaneous communication, with regard to some allusions or precedential facts (e.g., the eleventh of September) that can be unknown. What to do in the case of an interpreter? How to translate the missing piece? Theoretically, a lacunar element cannot be transferred. On the other hand, the gap should be filled by an interpreter, which is specified by broad interpretation. The choice of a tactic is formed according to established conditions and by the planned decision of an interpreter, or by a customer's demand for translation. 
To render lacuna, or remove lacuna, requires two methods of translation, cf.: the the vague element may be rendered by a shift (i) (transposition, substitution or modulation) or (ii) by means of the omission technique (deletion). Vinay claimed that these principles were actually the basis for "The Canadian School of Translation" (Vinay \& Darbelnet, 1958: 148).

Lacunae can be eliminated by either omission or shift (substitution, modulation or transposition). The omission is the 'deletion of lacuna', 'absence of translation' or by putting the specific element in italics without translation. A translation Shift is typically applied in this sphere (Anokhina \& Kobyakova, 2017: 34).

The source texts obviously present problems for any translator who has to render a text from a particular language or culture. Indeed, the cultural phenomenon can be lacunar to another culture. So the question is whether to preserve specifics by giving the italics of the lacunar artifact (e.g. tet-a-tet) without translating (1), to cut the lacuna off (2), or to compensate the lacuna by commentary or footnote. Anthony Pym describes at least four different strategies to render lacunae in his article "Multilingual Intertextuality in Translation". The theorist and a translator give a few examples on translation and nontranslation revealimg what could be described as translational or peri-translational: (1) source-language phrases are rendered at word level; (2) source-language cultural items are replaced with functionally equivalent target-language items; (3) source-language phrases are explained; and (4) new material is added. The important point is that all four strategies work in unison, producing a text that is restricted to neither one language, nor the other. The result is a commentary on political correctness in at least two cultures (Pym, 1996: 209).

The task of translation is to ensure cross-language communication in the text created in the language of the recipient, or "target language"; which can act as a complete replacement of the original text, or "source language" in the functional, structural and content relationships. It can be challenging because the texts as part of the same message are based on different language systems with units that do not match, either in form or in content. The possibility of the translated patterns is determined by the ability of multilingual texts to be communicatively equivalent in the process of information exchange. The equivalence of the original and target text is achieved in case when the translation reproduces the original content transmitted by means of another language. Translator has to accurately convey the structure and the content in the original text without deliberate structured transformations. A translation can be defined as a kind of linguistic mediation, in which the created text is communicatively equivalent to the original, and its equivalence is evident in functional, contextual and structural levels (Burbekova, 2014: 122). Some translators claim that the translator changes some details in the structure of the text, which should be done only in order to convey the original content better (substitution). Others claim that clarification works better for lacunae elimination (footnote, remark or translator's notes).

In the Romanian tradition, G. Lungu Badea (2004) states that facing cultural transfer, the translator usually chooses between three main options, namely (1) to borrow the foreign term, (2) to use an existing word or collocation with a new sense, or (3) to use an existing word complying as much as possible with the suggested pragmatic functions (Badea, 2004: 108). 
Vlahov and Florin (1980: 92) make a general differentiation between word transcription and any kind of translation, the latter being divided into several categories: (1) transcription of realia; (2) translation of realia; (3) Introducing a neologism (calques, semi-calques, assimilations, semantic neologisms); (4) approximate translation (the use of hyponyms/hypernyms; functional analogies; description, explication, interpretation); (5) contextual translation (zero translation (omission) with the transfer of the unit's meaning in the context) (Shiryaeva, 2014: 885). The ability to choose the correct translation technique is an indispensable skill (Zainudin, 2012: 328).

The culturally marked words are called lacunae and can be rendered differently. One of the first taxonomies was suggested in 1953 by A.V. Fedorov, who proposed to render lacunae by (1) full or partial transliteration, or transcription; (2) creation of a new word, or word combination on the basis of existing language elements and morphological relations and (3) using a unit which fulfils a similar (even though not identical) function in the target language (Fedorov, 1953: 139).

Today, many translators referring to translation techniques regard (1) semantic equivalence. Thus, within the context, the word "outcome" may be translated as "resulted". In regard to idiomatic equivalence (2), idiomatic expressions necessitated adaptations. Regarding cultural equivalence (3), some items may be slightly modified in translation for greater coherence to the cultural context of the target language population, which is sufficient to transmit the idea. Regarding conceptual equivalence (4), there is need for conceptual adaptations (Oliveiraa, 2014: 303).

The different types of discourse tend to demonstrate both (i) substitution and (ii) clarification in fiction, scientific discourse, media preserving lacunar information or eliminating it by footnote.

\section{Methods of lacunae identification in lacunology and translation studies}

The methodology of lacunae identification is based on the cognitive scenario "Language, Speech and Cognition". It helps to compile the corpus-based lacunicon in which three main endozones can be identified. Thanks to that it has become possible to verify the terms and notions of lacunology (lacunar lemmas) by singling out a semiotic-linguistic endozone (e.g., omission, reduction, elision, the absent categories in contrasting languages), a speech-translational endozone (e.g., syntagmatics, cultural and translation gaps, culture bumps, pauses, the silence effect, ellipses, etc.), and a cognitive synergetic endozone (allusions, decoding, conceptual ellipsis, etc.).

According to the above stated approach, lacunae can be seen as zero forms (e.g., zero pronoun), absent categories (the "erased category of plurality") (e.g., scissors), pauses (the silent effect in communication) and lacking or unknown information, or other lacunar models (decoding).

The experimental methodology of locating lacunar lemmas is carried out on a corpus-based study. It comprises quantitave methods of analysis for basic words of lacunology (the core of the lacunicon), discourse study for rare words search (hapaxes) (the part of a linguistic lacunicon corpus). It helps to find lacunae as rare 
words or hapaxes with rare (less than <1) frequency in a selected text which can be treated as a subcorpus of a corpus-based lacunicon.

In order to detect gaps in translation (non-translated elements), tertium comparationis is used, which helps to decode the lacuna through the original and translate using another parallel, or several copies of the translation. To detect the effectiveness of duplication one compares several duplicate versions that make specific parameters, ethno-cultural or other features that are differently accentuated in the translation versions. The analysis of the relayed versions of the translation allows for more precise identification and elimination of lacunar elements - verbal and nonverbal, subject or conceptual units.

An effective method is to detect gaps in parallel enclosures of translated texts. Comments and extra-text links are very informative to create a registry of lacunar units. Any nomination given for the concordance analyzer has a different representation in different discourses. The degree of use of the term "lacuna" (as well as other nominations for the analyzer) is calculated by the frequency list tool in the COCA.

The use of casing data is a modern trend in linguistics, and gives preference to this way of obtaining data for analysis. The modern features of Internet resources are vast and need to be streamlined for comfortable use.

COCAs contain different numbers of "lacuna" nominations in different discourses, for example: academic discourse / academic core (87), artistic discourse / fiction (41), press / magazine (12), show a high level of use of the term "lacun" when the spoken discourse / spoken (0) and media discourse / news (10) record the least amount of use of the nomination.

Translations of legal, medical, scientific and technical discourse / legal, medical, science $(65 \%)$ show the largest repeated automatic translation due to a high degree of reuse of cliché expressions and typically unambiguous blocks of text. Art discourse / fiction and the translation of other contextually dependent texts (35\%) are not effective because of the lack of case-based material in the database. At the time, these types cannot be efficiently translated using automatic translation systems and require translation and editing to a greater extent than informative non-significant texts.

The MonoConcEasy text analyzer can be used to search and analyze lacunae. This program has broad functionality for analysis and is used to search for hapaxes and rare words within texts, concordances and enclosures (by Michael Barlow). In order to find the text in the text / concordance / body, you must specify the minimum and maximum frequency of the use of a word that is numerically equal to one.

Hapaxes (rare words) in the investigated data are contextual gaps which are found in the analysis of texts using frequency programs. Few frequencies are rarely used by the author of the word $(0.0006 \%)$ within this body, for example: amiableness, amid, amidst, amiss, amity, housebreaking, mediocre, misery, recollecting. repentance, respect, robin, sacrifice (MonoConcEasy).

Hapaxes can be found in the analysis of texts using frequency programs. Lowfrequency ones are rarely used by the author of the word $(0.0006 \%)$ within this body, for example: amiableness, amid, amidst, amiss, amity, housebreaking, mediocre, misery, recollecting. repentance, respect, robin, sacrifice (MonoConcEasy). The search for hapaxes may be done in a text or in parallel and multi-language corpora. There are new approaches for extracting rare words (e.g. myometrium, lysergide, hyoscyamus, 
lysichiton, brassicaceae, yarrow, spikemoss, leiomyoma, ryegrass) from translations among aligned comparable documents which is applicable across different training and testing language pairs, making it possible to find rare word translations even for languages without training data (Prochasson \& Fung 2011: 1334).

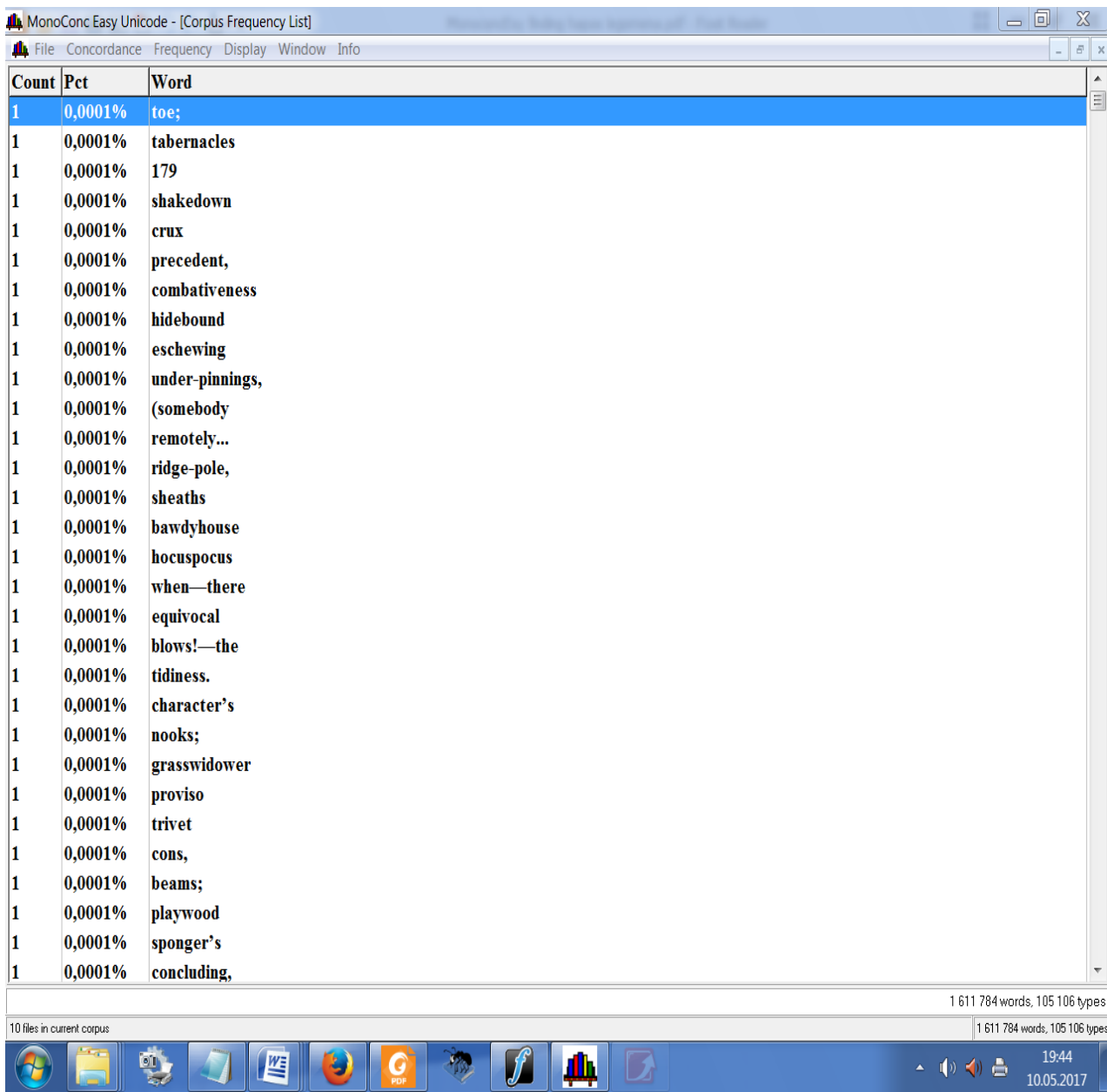

Figure 2. Hapaxes search by MonoConcEasy

\section{Conclusion}

The lacunae (gaps) are often associated with the problem of translation difficulties that can be attributed to a lack of room, not in the target culture, but to lacunae in the source culture. There are some specific elements in the national picture of the world that can not correspond to one-word notion, the lexical equivalent or have some differences compared to the other language.Lacunae are a manifestation of the incommensurability, incongruency, mismatch of cultures and languages. The lacunae arise in comparison, in contact with other 
languages and cultures. Lacunae are the elements of national-specific nature, they have are often interpreted as incomprehensible, unusual, exotic, strange, unfamiliar, and enigmatic elements.

The phenomenon to describe all types of lacunae is called lacunarity. The linguistic term to embrace the cross-linguistic lacunarity, cognitive lacunarity and the proper linguistic lacunarity (lacunae of the language and speech) is called the lacunicon. The terms of lacunicon include the term "lacuna" corresponding to the term "gap" in the international linguistics. Further research in the linguistic verification of lacunae will be oriented towards finding lacunae, hapaxes in texts and corpus data.

\section{References}

Anokhina, Tetiana. 2015. "Lacunae in terms of scholars: the brief overview." Filologychni traktaty 7, no. 1: 7-11.

—. 2013a. "The linguistic lacunicon: cognitive mapping in schemes and terms." Journal of Education, Culture and Society (Wroclaw, 2013): 166-175. doi: doi10.15503/jecs20131-166-174

—. 2013b. "The development of lacunology thesaurus in prototerms of lacunicon". Paper presented at The 2nd International scientific conference "Development of Scientific Thought in the 21st Century: Problems and Perspectives", Ryga, April, 2013: 38-41.

Anokhina, Tetiana, and Kobyakova, Iryna. 2017. "Something is lost: lacking information in translation". In Edges of scientific creativity: International collection of scientific papers (collective monograph) of the GISAP project participants, 32-34. London, October 18-24. https://gisap.eu/ru/node/138119

Auster, Paul. 1986. The New York Triology. City of glass. Ghosts. The locked room. London: Penguin books.

Badea, Georgiana L. 2004. Teoria culturemelor, teoria traducerii. Timisoara: Editura Universitatii de Vest.

Burbekova, Saule, Nurzhanova, Aisulu. 2014. "Problems of Translation Theory and Practice: Original and Translated Text Equivalence". Procedia - Social and Behavioral Sciences 136 (9): 119-123.

Burt, George. 2003. "Epigenetic change: new from the seeds of the old". Strategic Change 12(7). Stirling: Wiley-Blackwell: 381-393.

Cook, Malcolm E., Lehnert Wendy G., Mcdonald David D. 2017 “Conveying implicit content in narrative summaries". Proceedings of the 10th international conference on computational linguistics and 22-nd annual meeting on Association for Computational Linguistics. Stanford, California, July 2-6: 5-7.

Fedorov, Andrei. 1953. Vvedenije v teoriju perevodovedenija. Moscow: Izdatelstvo literatury na inostrannyh jazykah.

Florin, Sider. 1993. "Realia in Translation”. In Translation as Social Action: Russian and Bulgarian perspective, Zlateva Palma, 122-128. London: Routledge.

Frank, Andrew U. 2006. "Distinctions Produce a Taxonomic Lattice: Are These the Units of Mentalese?" In: International Conference on Formal Ontology in Information Systems, 166-181. Baltimor: IOS Press.

Gak, Vladimir. 1977. The Contrasting Lexicology. The work performed on the material of the French and Russian languages. Moscow.

Glazacheva Natalia. 2005. "Lacunae and the theory of intercultural communication". In Lacunae in the language and in speech: the proceedings of the scientific papers 2: 32-34. Blagoveshensk: the Publishing House of the BSPU. 
Intan Safinaz Zainudin, Norsimah Mat Awal. 2012. "Translation Techniques: Problems and Solutions". Procedia - Social and Behavioral Sciences 59: 328-334, October 172012.

Oliveiraa, Liszt Palmeira, Themis Moura Cardinotb, Letícia Nunes Carreras Del Castilloa, Marcelo Cavalheiro Queirozc, Giancarlo. 2014. "Translation and cultural adaptation of the Hip Outcome Score to the Portuguese language.” Revista Brasileira de Ortopedia (English Edition) 9 (3): 297-304, May-June.

Prochasson, Emmanuel, and Fung, Pascale. 2011. Rare word translation extraction from aligned comparable documents. In Proceedings of the 49th Annual Meeting of the Association for Computational Linguistics: Human Language Technologies 1: 1327-1335.

Pym, Anthony 1993. Lacunae and uncertain limits in in Australian culture, with suggestions on their translation into Spanish, edited by Kathleen Firth and Susan Ballyn, 2737. Barcelona: Universitat de Barcelona.

—. 1996. Multilingual Intertextuality in Translation, edited by Beatriz Penas Ibáñez. The Intertextual Dimension of Discourse: 207-218. Universidad de Zara-goza. http://usuaris.tinet.cat/apym/on-line/translation/multilingual.pdf 1: $65-77$.

Shopen, Tim. 1973. Ellipsis as Grammatical Indeterminacy. Foundations of Language 10, no.

Sorokin, Yurii, Markovina Irina, Kryukov Aleksei. 1988. Etnopsycholinguistics. Moscow: Nauka.

Sternin, Iosif. 2004. Contrastive linguistics. The problems of theory and methodology of investigation. Voronezh: Istoki publishers.

Sternin, Iosif, and Flekenshtein Kurt. 1989. Studies on contrastive lexicology and phraseology: Textbook. benefit: in 2 parts. Halle.

Szerszunowicz, Joanna. 2015. "Lacunarity, lexicography and beyond: integration of the introduction of a linguo-cultural concept and the development of L2 learners' dictionary skills". Lexicography, 2(1): 101-118. DOI: doi.org/10.1007/s40607-015-0015-6

Valentina Shiryaeva, Georgiana Lungu Badea. 2014 "Subtitling: The Transfer of Culture-specific Words in a Multidimensional Translation". Procedia - Social and Behavioral Sciences 149: 883-888, September 5.

Venuti, Lawrence. 1995. The Translator's Invisibility. A History of Translation. London and New York: Routledge.

Vinay, Jean-Paul and Jean Darbelnet. 1958. Stylistique comparée du français et de l'anglais: méthode de traduction. Nouvelle édition revue et corrigée, Didier, Paris. otnošenija.

Vlahov, Sergei, Florin, Sider. 1980. Neperevodimoje v perevode. Moscow: Meždunarodnyje

Webster Noah, Goodrich, Chauncey A., Porter, Noah. 1848. Webster's New World Dictionary of the American language. Springfield, Mass.: G. and C. Merriam.

Zainudin, Intan Safinaz, Awal, Norsimah Mat. 2012. Translation Techniques: Problems and Solutions. Procedia - Social and Behavioral Sciences 59: 328-334, October 17.

Zhelvis, Vladimir. 1977. On the issue of the character of the of English-Russian lacunae. The Research on Problems of the Speech Communication. Moskow: Nauka publishers.

Zhelvis, Vladimir, Markovina, Irina. 1979. The experience of systematization of English-Russian lacunae. The Research on Problems of the Speech Communication. Moskow: Nauka publishers. 